Full Length Article

\title{
UV laser annealing of Diamond-Like Carbon layers obtained by Pulsed Laser Deposition for optical and photovoltaic applications
}

\author{
F. Stock ${ }^{a, *}$, F. Antoni ${ }^{a}$, L. Diebold ${ }^{a}$, C. Chowde Gowda ${ }^{a}$, S. Hajjar-Garreau ${ }^{b}$, D. Aubel $^{\mathrm{b}}$, \\ N. Boubiche ${ }^{\mathrm{a}, \mathrm{c}}, \mathrm{F}$. Le Normand ${ }^{\mathrm{a}}$, D. Muller ${ }^{\mathrm{a}}$ \\ a ICube, D-ESSP, 23 rue du Loess, 67037 Strasbourg, France \\ ${ }^{\mathrm{b}}$ Institut de Science des Matériaux de Mulhouse, IS2M-CNRS - UMR7361, 15 rue Jean Starcky, 68057 Mulhouse Cedex, France \\ ${ }^{\mathrm{c}}$ Laboratoire d'étude physico-chimiques des matériaux (LEPCM), Faculté des sciences de la matière, Dép. de physique, Université Batna1, 05 Avenue Chahid Boukhlouf., \\ Batna 05000, Algeria
}

\section{A R T I C L E I N F O}

\section{Keywords:}

Pulsed Laser Deposition (PLD)

Diamond-Like Carbon (DLC)

Optoelectronic properties

Laser surface annealing

\begin{abstract}
A B S T R A C T
One of the biggest challenge in optoelectronic devices is the necessity to provide a viable and reliable alternative to Transparent Conducting Oxide (TCO) and especially to Indium Tin Oxide (ITO). Graphene is a widely studied material and one of the best alternative to be used as conductive and transparent electrodes. It is well known that the difficulty to transfer graphene on another substrate is a serious limitation for its use on large scale devices. In this work, we explore Diamond-like Carbon (DLC) thin films prepared by Pulsed Laser Deposition (PLD) to be used as substrate for graphene-like layers. DLC thin films are excellent candidates due to their visible-range transparency being also a very good electrical insulator. Transmission measurements show the UV opaque character of the DLC layers, independently to the experimental parameters used to produce them. Thus, top-hat UV laser surface annealing can strongly modify the DLC thin film structure in order to bring conductivity to the first atomic layers. Raman spectroscopy and X-ray photoemission spectroscopy permit to confirm the graphitic character of the DLC surface. Optimizing PLD as well as laser annealing parameters is explored in detail in order to obtain comparable performances (conductivity and transparency) to ITO typical properties. Moreover, using a full-based laser process offers a complete compatibility with all technical steps of the microelectronic domain.
\end{abstract}

\section{Introduction}

The most common transparent conductive material used nowadays in optoelectronic and photovoltaic devices is Indium Tin Oxide (ITO). This one owns very high transparency in the visible range and very high conductivity over large areas $[1,2]$. In the forthcoming years, we will face an increasing problem due to the indium rarefaction. Moreover, the impossibility of correctly recycling ITO is doomed to be a problem for the optoelectronic and photovoltaic components. A suitable alternative to this metallic oxide is needed. The hereby proposed solution is fully compatible with all the materials and processes used in micro and optoelectronics.

The Diamond-Like Carbon (DLC) is a material introduced nowadays in photovoltaic modules as encapsulation and protective anti-reflexion coating $[3,4]$. DLC is an amorphous form of carbon used as a low-cost substitute to diamond. This material is a very good electrical insulator due to kinship with diamond [5-7]. DLC is constitute by a mixture of $\mathrm{sp}^{2}$ and $\mathrm{sp}^{3}$ bounded carbon atoms [8]. $\mathrm{Sp}^{2}$ is the stable binding corresponding to graphitic material whereas the $\mathrm{sp}^{3}$ is the metastable binding related to the adamant character which is formed under special conditions of temperature and pressure. The amount of each carbon hybridisation and correlated properties are directly related to the process used to elaborate DLC [9-11]. Another important property that DLC share with diamond is a high visible and infrared range transparency. Combined to its insulating property drives to use DLC as an insulating transparent substrate. We use Pulsed Laser Deposition (PLD) of carbon to grow DLC thin films. Those films possess advantageous properties directly depending on the deposition process parameters [8,12-14]. Moreover, PLD allows while working under high vacuum, to obtain high purity, oxygen and hydrogen-free DLC layers. As we previously demonstrated, the $\mathrm{sp}^{2}$ and $\mathrm{sp}^{3}$ amount ratio is highly depending on the laser wavelength and the energy density used to ablate the carbon. Those parameters condition the optical and electrical DLC properties [15].

Graphene is another form of carbon. Its two-dimensional structure only constituted by a single layer of $\mathrm{sp}^{2}$ bounded carbon atoms, is

\footnotetext{
* Corresponding author.

E-mail address: fstock@unistra.fr (F. Stock).
} 
organized in a honeycomb pattern. Well studied in the last years, graphene has a very high electron mobility assuming an excellent electrical conductivity $[16,17]$. Moreover, graphene has a very low optical absorption approaching $2.3 \%$ per layer and can also be considered as a transparent electrical conductor. Despite many elaboration methods like chemical vapour deposition, graphite exfoliation or aerosol, it is nowadays very hard to transfer a graphene layer or large graphenic areas for electronic applications. Combining the properties of DLC and graphene could be an innovative solution to obtain a transparent conductive material directly set on an insulated transparent substrate.

Our original approach to obtain a pure carbon transparent conductive material is based on a two steps process. In first, we use PLD to grow a high purity DLC thin film. In a second step, the layer is exposed to an UV laser surface treatment. Indeed, compared to crystalline diamond, DLC present a very high UV opacity. Therefore, the use of deep UV excimer laser annealing at very low energy drives to modify only the DLC top structure by interacting only with the very first atomic layers of the film. In addition, laser annealing is only possible with PLD produced DLC due to the absence of hydrogen in the layers. Surface treatments will force carbon atoms to be reorganized in pure $\mathrm{sp}^{2}$ forming top graphitic layers. Those few surface layers will bring the requested conductivity. This full process, only based on laser technology, offers therefore a complete compatibility with standard microelectronic and photovoltaic processes.

\section{Experimental details}

\subsection{Pulsed laser deposition of DLC}

We use a standard PLD setup to perform our DLC depositions. A laser is focused with a $45^{\circ}$ incidence angle on a high purity graphite target $(99.999 \%)$ placed into a high vacuum chamber (residual pressure less than $10^{-8}$ mbar). When the laser hits the target, the matter is ablated perpendicularly to its surface and collected on a substrate set parallel in front of it. The target-substrate distance is kept constant and set to $5 \mathrm{~cm}$ in order to obtain a homogeneous sample coverage over $1 \times 1 \mathrm{~cm}^{2}$. The target is rotating at $1 \mathrm{rpm}$ in order to avoid crater formation by successive ablation at the same position. The excimer laser (LAMBDA PHYSIK Compex 201 working with a $\mathrm{KrF}$ gas mixture corresponding to a $248 \mathrm{~nm}$ wavelength) produces $600 \mathrm{~mJ}$ pulses (25 ns duration) at repetition rates from 1 to $10 \mathrm{~Hz}$. After optimal focusing and taking in account all optical component energy losses, we reach ontarget fluences tuneable from 2 up to $15 \mathrm{~J} / \mathrm{cm}^{2}$. As shown in previous studies $[15,18,19]$, DLC thin films are deposited at room temperature to obtain the highest adamant character. The layers were mainly deposited on oxidized silicon $\left(\mathrm{SiO}_{2}(63 \mathrm{~nm}) / \mathrm{Si}\right.$ substrates of $\left.1 \times 1 \mathrm{~cm}^{2}\right)$. For optical measurements only, layers were deposited on quartz substrates.

\subsection{Laser surface annealing}

To perform laser annealing on the DLC layers, the $248 \mathrm{~nm}$ excimer laser beam passes through an optical homogenizer in order to obtain a $1 \times 1 \mathrm{~cm}^{2}$ top-hat beam (better than $1 \%$ beam homogeneity). A treatment chamber, equipped with a quartz window (for the UV laser input) was built in order to anneal samples under vacuum or neutral gas atmosphere. With this setup, the laser beam reaches a $5 \times 5 \mathrm{~mm}^{2}$ homogeneous size, allowing to work with fluences between 0.05 and $0.36 \mathrm{~J} / \mathrm{cm}^{2}$ (low enough to avoid overpassing the carbon ablation threshold, which is higher than $1 \mathrm{~J} / \mathrm{cm}^{2}$ ). In order to treat large areas, the whole chamber is fastened to a XYZ table. The chamber is moved under the homogeneous $5 \times 5 \mathrm{~cm}^{2}$ laser beam. A special designed software calculates the different sample positions insuring a full area covering. Homogeneity is guaranteed by the use of a $90 \%$ overlap between each defined position permitting large surface annealing (up to $20 \times 20 \mathrm{~mm}^{2}$ ), only limited by the chamber input window dimension.
The laser is triggered by the software and operates at a $10 \mathrm{~Hz}$ frequency.

\subsection{Characterization techniques}

To perform structural analyses and quantify the changes induced by the laser annealing, we use X-ray photoemission spectroscopy (XPS). Measurements have been performed at two different incidence angles on a $4 \mathrm{~mm}^{2}$ surface with a $1486.6 \mathrm{eV}$ beam energy. In fact, with a $20^{\circ}$ angle (named here as "normal incidence"), we obtain spectra corresponding to a $9.1 \mathrm{~nm}$ ( $\pm 0.35 \mathrm{~nm}$ ) depth integration as deduced by simulation, whereas with a $60^{\circ}$ angle (grazing analysis), only $4.8 \mathrm{~nm}$ $( \pm 0.3 \mathrm{~nm}$ ) were analysed. Therefore, compared measurements permit to set difference between surface and in-depth layer analysis. Spectra are analysed by focusing on C1s peak of carbon. The deconvolution of this peak allows quantifying the relative $\mathrm{sp}^{2}$ and $\mathrm{sp}^{3}$ bindings amount due to a slight difference in binding energy levels. [11,20,21]. Insuring a very low energy levels shift (less than $1 \%$ ) between all our samples, we quantify $\mathrm{sp}^{2}$ and $\mathrm{sp}^{3}$ contribution values with a very good precision $( \pm 0.5 \%)$. Measurements also show a very low amount of oxygen contamination on the layer surfaces. This contribution is negligible and we will not be considered in the discussion.

Conductivity values are obtained with the 4 probes Hall effect measurement in a Van der Pauw configuration. Each probe is set in a corner of the $1 \times 1 \mathrm{~cm}^{2}$ sample directly on the DLC surface. It is important to notice that conductivity measurements confirm the high insulting character of untreated DLC films.

Transmittance measurements are performed with a UV-visible spectrometer from 250 up to $850 \mathrm{~nm}$ with a scanning speed of $30 \mathrm{~nm}$ per minute. Deposition are specially done on quartz substrates to provide high UV-visible transparency. The initial quartz sample transmission spectrum was also acquired. The single DLC signal was extracted by subtracting the quartz substrate response. After laser annealing, we use the same method to obtain the single response of treated DLC.

\section{Results}

\subsection{DLC as deposited}

We have deposited $20 \mathrm{~nm}$ of DLC at a fluence of $5 \mathrm{~J} / \mathrm{cm}^{2}$. A previous study [15] has shown that for these parameters, DLC layers contains $60.9 \% \mathrm{sp}^{3}$ bounded atoms and $39.1 \% \mathrm{sp}^{2}$, homogeneously distributed in the entire layer. Transmittance measurements reveal a good transparency in the visible range (approaching $80 \%$ at $800 \mathrm{~nm}$ for a $20 \mathrm{~nm}$ thick layer). This value is sinking down for shorter wavelengths (reduced to $33 \%$ at $248 \mathrm{~nm}$ ). Measurements are in accordance with the light brown colour of the samples.

\subsection{Laser annealing experiments}

The annealing process was performed in first attention under air atmosphere. These preliminary experiments show many defects over the entire DLC surface. In fact, when the laser hits the DLC surface, the energy breaks the existing bindings between carbon atoms. In presence of oxygen, those atoms react and form monoxide and dioxide carbon. This reaction results in the DLC layer delamination associated with a full transparency loss. Therefore, we avoid contact with air during the annealing process and work systematically under a neutral gas pressure (0.5 mbar of argon).

We performed various treatments on a series of $20 \mathrm{~nm}$ thick $\mathrm{KrF}$ $\left(5 \mathrm{~J} / \mathrm{cm}^{2}\right)$ deposited DLC. We compared various number of shots $(100$, 1000 and 10,000$)$ at various fluences $\left(0.1\right.$ up to $\left.0.36 \mathrm{~J} / \mathrm{cm}^{2}\right)$ in order to analyse the impact on the DLC surface and to find an optimal set of parameters. We observed that all treatments made at a fluence equal or higher than $0.3 \mathrm{~J} / \mathrm{cm}^{2}$ induce a large number of defects formation, even for 100-1000 shots. We investigated those defect-affected areas with 


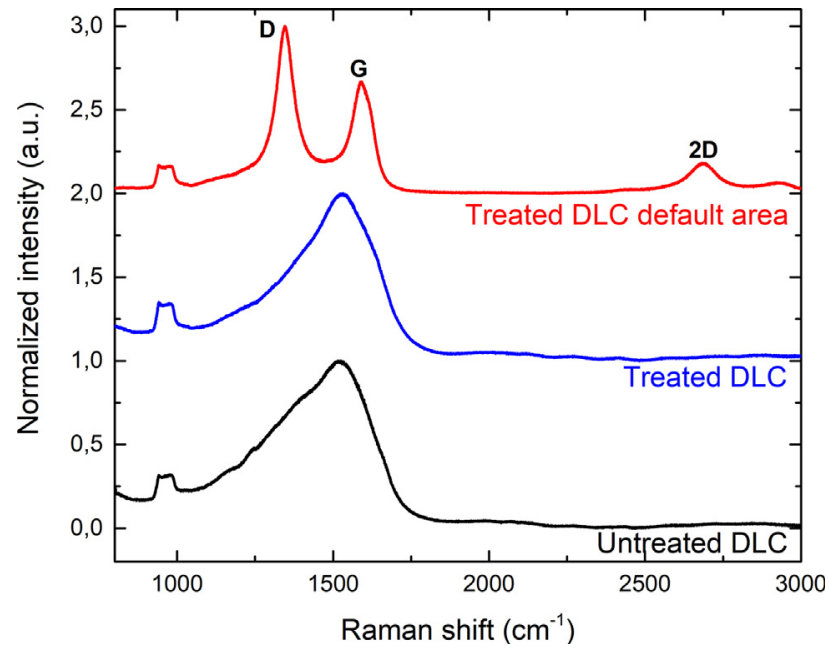

Fig. 1. Raman spectra of DLC $\left(\mathrm{KrF}-5 \mathrm{~J} / \mathrm{cm}^{2}-20 \mathrm{~nm}\right)$ before and after laser annealing.

Raman spectroscopy (JOBIN YVON LabRam Aramis with a $532 \mathrm{~nm}$ excitation) and observed graphitic spectra, characterized by the presence of clear distinguished D and $G$ peaks coupled with a low intensity 2D peak (Fig. 1). As observed for treatments under air condition, we see that those defects lead to a layer disintegration correlated with a loss of transparency. We think that surface captured residual oxygen (and other impurities) drives to these defects formation. For laser annealing performed at $0.1 \mathrm{~J} / \mathrm{cm}^{2}$ and $0.2 \mathrm{~J} / \mathrm{cm}^{2}$, only sparsely very small defects are visible on the surface, even with a high number of 10,000 shots. Only a tiny colour change can be seen on the surface, corresponding to a slight DLC darkening. Raman spectroscopy of these treated areas do not show any significate change compared to untreated DLC (Fig. 1). We assume that Raman spectroscopy is in our case, unable to catch surface modification due to the DLC transparency at $532 \mathrm{~nm}$, driving then to a full DLC thickness analysis. We made structural characterization to investigate more precisely the changes induced by the surface annealing.

These preliminary experiments show that, to keep the transparency of the DLC layer after laser surface annealing, we have to keep the fluence under a threshold of $0.2 \mathrm{~J} / \mathrm{cm}^{2}$. By overpassing this fluence, large graphitic areas are formed and the surface layer is highly affected.

\subsection{XPS analyses}

We extract from XPS spectra the $\mathrm{sp}^{2}$ and $\mathrm{sp}^{3}$ bindings ratio of samples treated at $0.1 \mathrm{~J} / \mathrm{cm}^{2}$ and $0.2 \mathrm{~J} / \mathrm{cm}^{2}$ with 1000 (Fig. 2a) and 10,000 shots (Fig. 2b) respectively. It is clearly observed that for an increasing fluence, the $\mathrm{sp}^{2}$ binding amount at the surface is increasing, varying from $39.1 \%$ for an untreated DLC up to $59.7 \%$ for a treatment at $0.2 \mathrm{~J} / \mathrm{cm}^{2}-1000$ shots. We also observe that the annealing performed at 10,000 shots, the $\mathrm{sp}^{2}$ concentration is even higher reaching $68 \%$ at $0.2 \mathrm{~J} / \mathrm{cm}^{2}$. We can conclude that both increase of laser annealing fluence and number of shots drives to an increase of the graphitic bindings present at the surface of the DLC, correlated with a $\mathrm{sp}^{3}$ bindings decrease.

Moreover, this increase is also observed for deeper layer analyses. Indeed, we observe the same behaviour as for grazing analyses but with a lower $\mathrm{sp}^{2}$ percentages. A direct comparison gives a $68 \% \mathrm{sp}^{2}$ amount for grazing analysis and a $59.8 \% \mathrm{sp}^{2}$ amount for a normal angular analysis at $0.2 \mathrm{~J} / \mathrm{cm}^{2}-10,000$ shots. For each set of annealing parameters, the $\mathrm{sp}^{2}$ concentration is higher at the surface. In accordance to the fact that XPS normal and grazing analyses integrate bindings present at the surface, we can conclude that the laser annealing mostly affects the surface of the DLC (over a few nanometers only), changing it

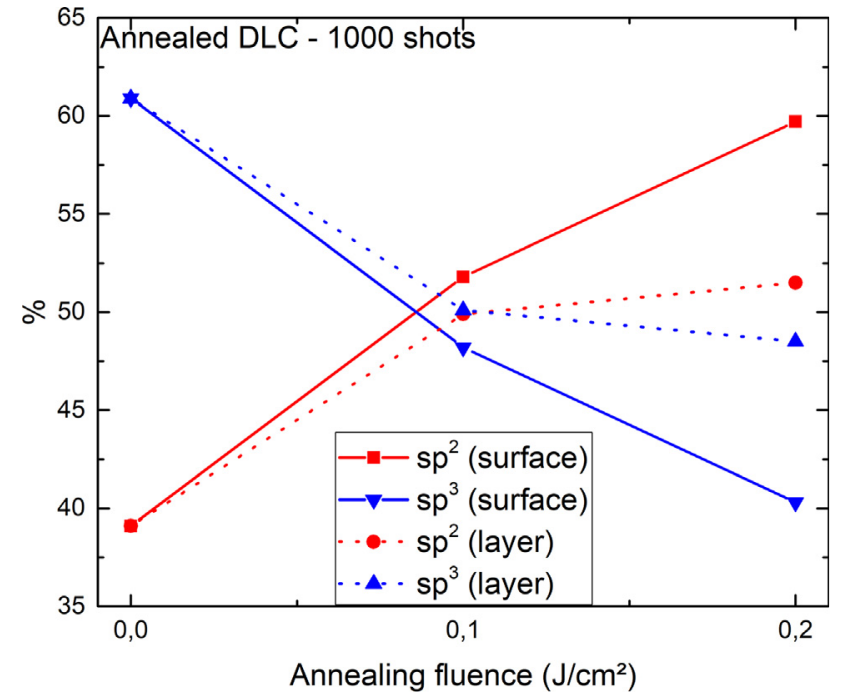

Fig. 2a. Hybridization ratio for DLC $\left(\mathrm{KrF}-5 \mathrm{~J} / \mathrm{cm}^{2}\right)$ annealed at different fluences for 1000 shots (Point at $0 \mathrm{~J} / \mathrm{cm}^{2}$ corresponding to an untreated DLC).

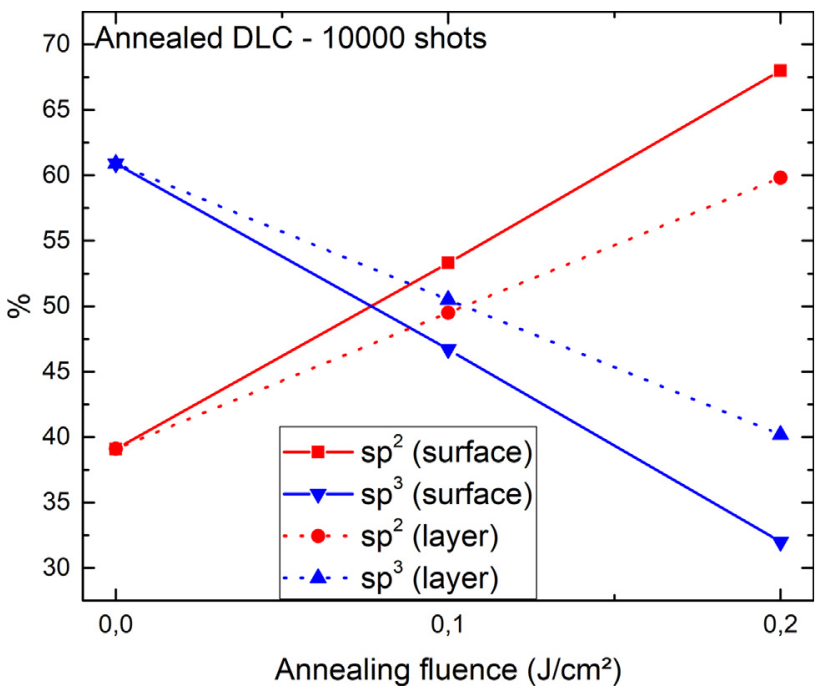

Fig. 2b. Hybridization amount for DLC $\left(\mathrm{KrF}-5 \mathrm{~J} / \mathrm{cm}^{2}\right)$ annealed at different fluences for 10,000 shots. (Point at $0 \mathrm{~J} / \mathrm{cm}^{2}$ corresponding to an untreated DLC).

into a pure graphitic cover layer.

\subsection{Conductivity measurements}

We measure a very low conductivity $\left(2.13 \times 10^{-3}(\Omega \mathrm{cm})^{-1}\right)$ for the sample annealed at $0.1 \mathrm{~J} / \mathrm{cm}^{2}$ with 10,000 shots. This conductivity is drastically increasing as soon as the laser annealing fluence is raised, reaching $5 \times 10^{4}(\Omega \mathrm{cm})^{-1}$ for a $0.2 \mathrm{~J} / \mathrm{cm}^{2}$ fluence (Fig. 3). For a treatment performed at $0.2 \mathrm{~J} / \mathrm{cm}^{2}$ with 1000 shots, a relatively high conductivity is also measured $\left(2.91 \times 10^{3}(\Omega \mathrm{cm})^{-1}\right)$. It means that either the annealing fluence and the number of laser shots directly influence the layer's conductivity. These measurements are correlated with the increase of the $\mathrm{sp} 2$ at the top of the DLC corresponding to a graphitic behaviour.

\subsection{Transmittance measurements}

We present in this part (Fig. 4) results obtained on a $\mathrm{KrF}$ deposited DLC $(20 \mathrm{~nm})$ at $5 \mathrm{~J} / \mathrm{cm}^{2}$ treated with a $0.2 \mathrm{~J} / \mathrm{cm}^{2}$ fluence for 1000 shots. A clear modification of the transmittance is observed after the 


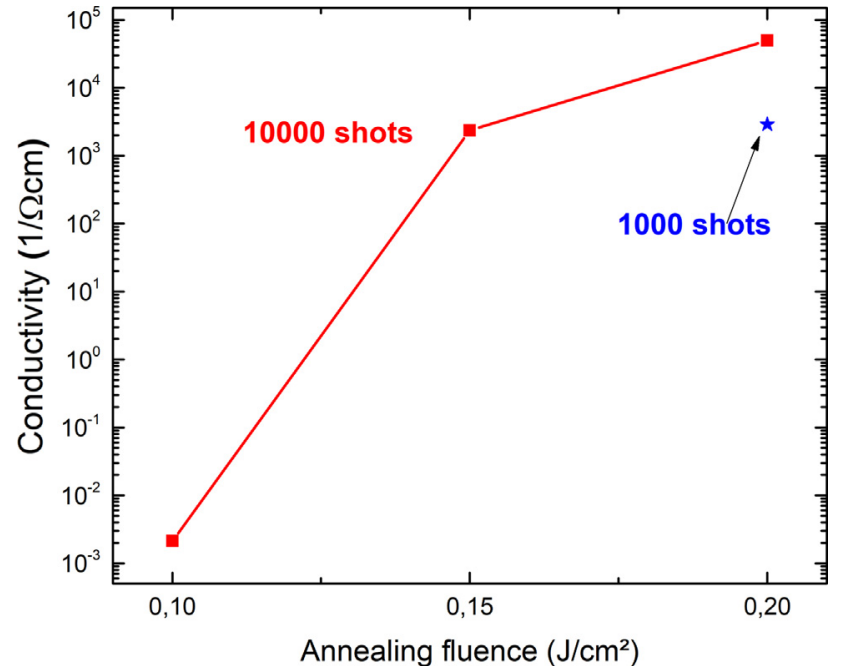

Fig. 3. Conductivity measurements on DLC $\left(\mathrm{KrF}-5 \mathrm{~J} / \mathrm{cm}^{2}\right)$ as a function of annealing fluences and shot numbers.

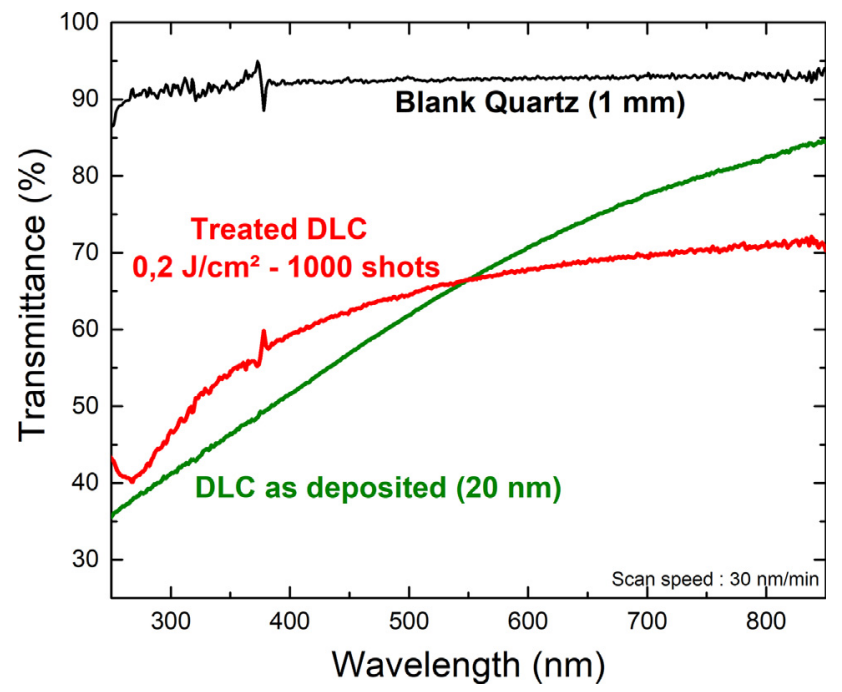

Fig. 4. Transmittance measurement of quartz, DLC $\left(\mathrm{KrF}-5 \mathrm{~J} / \mathrm{cm}^{2}-20 \mathrm{~nm}\right)$ and laser treated DLC $\left(0.2 \mathrm{~J} / \mathrm{cm}^{2}-1000\right.$ shots $)$.

annealing process. Approximatively $15 \%$ difference is measured near the infrared range $(70 \%$ of transmittance). This transmittance loss is less important when reaching lower wavelengths. A slightly higher transmittance (as for untreated DLC) is observed between 250 and $520 \mathrm{~nm}$. This peculiar behaviour is in accordance with the colour change detected visually on the sample, passing from a light brown before annealing to a light grey after it.

In addition, the laser treatment increases the $\mathrm{sp}^{2}$ amount in the DLC, so this point also correlates the fact that the transmittance is reducing after annealing due to the more graphitic character of the treated layer. We can conclude that the laser annealing only slightly modifies the DLC optical properties.

\section{Discussion}

Electrical measurements help to reduce the optimal parameters range, in the way that for a $0.1 \mathrm{~J} / \mathrm{cm}^{2}$ annealing, conductivity is clearly not satisfying. Withal a very high conductivity obtained for the $0.2 \mathrm{~J} /$ $\mathrm{cm}^{2}$ treatment and for a very high number of 10,000 shots, the DLC presents some sparsely small local surface defects. As discussed for airmade treatments, we suspect that for high annealing fluences combined with a high number of laser shots, the surface remaining residual oxygen (captured during the sample analysis or transfer) drives to defects formation. This is also applicable for other impurities that might be present on the surface. We believe that high-energy $\left(0.3 \mathrm{~J} / \mathrm{cm}^{2}\right)$ successive laser shots will progressively induce a DLC layer delamination. Therefore large graphitic defect areas are formed, leading to a strong surface roughness increase. Providing defect-free DLC or working under a better-controlled neutral gas atmosphere (or in high vacuum condition) shall be an important factor for working at fluences higher than $0.2 \mathrm{~J} / \mathrm{cm}^{2}$.

Optical measurements show that the laser treatments do not change the transmittance of the DLC so far. To improve this point, we should start by increase the original untreated DLC transmittance. A way to achieve this point is to reduce the DLC thickness. PLD has the advantage to provide a very precise control over the layer thickness. It is clearly possible to deposit only a few nanometers, obtaining therefore a very high transmittance in the visible range (over 90\%). The only limiting factor is the laser penetration depth in DLC. Indeed, XPS results help to determine an average value of $2.4 \mathrm{~nm}$ of fully graphitized material corresponding to an equivalent of seven atomic layers for laser annealed conductive samples. We have to take care of this point and keep enough matter to perform annealing in good conditions. Finding a compromise between the highest conductivity and high transparency by refining DLC deposition and treatment parameters is the key point for the optimization of our process.

\section{Conclusion}

We have demonstrated the possibility to obtain high conductive transparent DLC structures (approaching ITO performances) using a full laser combined process. High performance DLC deposited by PLD allows to obtain a very good transparent layer. A post UV-laser annealing is applied on the samples bringing surface conductivity while keeping a high value of transparency. Impacts of both fluence and number of shots on the electrical conductivity has also been studied. We show that increasing these parameters leads to a rise of $\mathrm{sp}^{2}$ bindings on a few carbon surface atomic layers correlated with a conductivity gain. A threshold fluence of $0.2 \mathrm{~J} / \mathrm{cm}^{2}$ has also been highlighted. Overpassing this fluence drives to the formation of large graphitic areas associated with a full transparency loss and layer delamination. A range of experimental parameters has been established in order to obtain the best performances.

Finally, it remains some possibilities to improve our layer performances regarding the deposition parameters. One way to improve the transparency is to reduce the DLC layer thickness. Another approach we consider, is to work with a DLC having a much better adamant character (deposited with a $193 \mathrm{~nm}$ wavelength, as we previously demonstrated). Finding a compromise between transparency and conductivity regarding initial DLC properties and tuning laser annealing parameters will be a major part of our future studies.

\section{Acknowledgement}

We specially want to acknowledge $\mathrm{Mr}$ ROQUES Stephane, $\mathrm{Mr}$ DIETRICH Florent and Mr MUGLER Florian for the technical support to carry on this study.

\section{References}

[1] Z. Chen, W. Li, R. Li, Y. Zhang, G. Xu, H. Cheng, Langmuir (November 2013).

[2] H. Kim, C.M. Gilmore, B. Chrisey, J. Appl. Phys. 86 (1999) 6451.

[3] F.J. Pern, Zh. Panosyan, A.A. Gippius, J.A. Kontsenvoy, K. Touryan, S. Voskanyan, Y. Yengibaryan, 31st IEEE PV Specialists Conference and Exhibition, Florida, January 2005.

[4] C.R. Lin, H.M. Chang, C.K. Chang, Int. J. Photoenergy (2013) ID 616263.

[5] E. Marotta, N. Bakhru, A. Grill, V. Patel, B. Meyerson, Thin Solid Films 206 (1991) $188-191$. 
[6] A. Grill, Thin Solid Films 355-356 (1999) 189-193.

[7] Z. Di, P.K. Chu, M. Zhu, R.K. Fu, S. Luo, L. Shao, M. Nastasi, P. Cheng, T.L. Alford, J.W. Mayer, M. Zhang, W. Liu, Z. Song, C. Lin, Appl. Phys. Lett. 88 (2006) 142108.

[8] J. Robertson, Mater. Sci. Eng. R 37 (2002) 129-281.

[9] A. LiBassi, A.C. Ferrari, V. Stolojan, B.K. Tanner, J. Robertson, L.M. Brown, Diamond Related Mater. 9 (2000) 771-776.

[10] F. Qian, R.K. Singh, S.K. Dutta, P.P. Pronko, Appl. Phys. Lett. 67 (1995) 21.

[11] R.M. Dey, S.B. Singh, A. Biswas, R.B. Tokas, N. Chand, S. Venkateshwaran, D. Bhattacharya, N.K. Sahoo, S.W. Gosavi, S.K. Kulkarni, D.S. Patil, Curr. Appl. Phys. 8 (2008) 6-12.

[12] A.A. Voevodin, M.S. Donley, Surf. Coat. Technol. 82 (1996) 199-213.

[13] G. Reisse, B. Keiper, S. Weissmantel, U. Falke, Appl. Surf. Sci. 127-129 (1998) 500-506.

[14] F. Xiong, Y.Y. Wang, V. Leppert, R.P.H. Chang, J. Mater. Res. (September 1992).
[15] F. Stock, F. Antoni, F. Le Normand, D. Muller, M. Abdesselam, N. Boubiche, I. Komissarov, Appl. Phys. A 123 (August 2017) 590.

[16] R.R. Nair, P. Blake, A.N. Grigorenko, K.S. Novoselov, T.J. Booth, T. Stauber, N.M.R. Peres, A.K. Geim, Science 320 (June 2008).

[17] S. Cho, M.S. Fuhrer, Center of Nanophysics and Advanced Materials, University of Maryland, 2008.

[18] S. Rey, F. Antoni, B. Prevot, E. Fogarassy, J.C. Arnault, J. Hommet, F. Le Normand, P. Boher, Appl. Phys. A 71 (2000) 433.

[19] Z. Nibennaoune, D. George, F. Antoni, S. Ahzi, D. Ruch, J. Gracio, Y. Rémond, Diamond Related Mater. 22 (2012) 105-112.

[20] H.Y. Dai, Y.Q. Wang, X.R. Cheng, C.Y. Zhan, N.K. Huang, Appl. Surf. Sci. 258 (2012) 5462-5466.

[21] H. Nakazawa, Y. Yamagata, M. Suemitsu, M. Mashita, Thin Solid Films 467 (2004) 98-103. 